\title{
Correlation and Path Coefficient Analysis for Yield Contributing and Quality Traits in Quality Protein Maize (Zea mays L.)
}

\author{
Ramesh Kumar*, R.B. Dubey, K.D. Ameta, Ram Kunwar, \\ Rajani Verma and Prashant Bisen
}
Department of Plant Breeding and Genetics, Maharana Pratap University of Agriculture and
Technology, Udaipur - 313001, Rajasthan, India
*Corresponding author

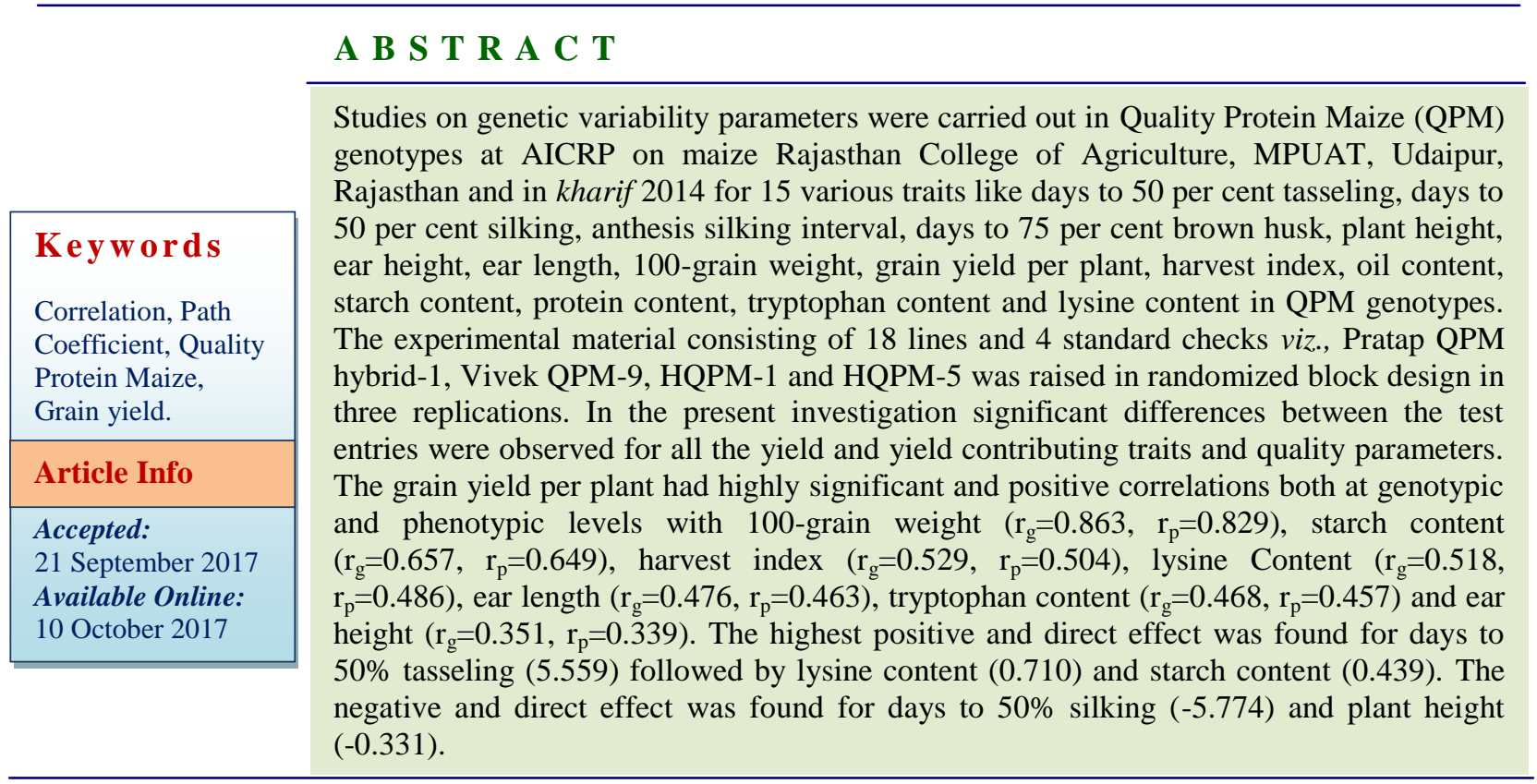

\section{Introduction}

Maize (Zea mays L.) is the third important cereal crop globally after wheat and rice (FAO, 2014). Maize being a $\mathrm{C}_{4}$ plant is physiologically more efficient, has higher grain yield and wider adaptation over wide range of environmental conditions which provides food, feed, fodder, fuel and severe as a source of basic raw material for a number of industrial products viz. protein, starch, oil, food sweeteners and bio-fuel etc. It grown over a range of agro climatic zones from $58^{\circ}$
$\mathrm{N}$ to $40^{\circ} \mathrm{S}$, from below sea level to altitudes higher than $3000 \mathrm{~m}$, and in areas with 250 $\mathrm{mm}$ to more than $5000 \mathrm{~mm}$ of rainfall per year (Downswell et al., 1996).

Because of very wide utilization of maize, the main goal of all maize breeding programs is to obtain new inbred and hybrids that will outperform the existing hybrids with respect to a number of traits. In working towards this goal, particular attention is paid to grain yield 
as the most important agronomic characteristic (Pandey et al., 2017).

Grain yield is a complex quantitative trait that depends on plant genetics and its interaction with environmental conditions (Khatab et al., 2016). To determine such relationships, correlation analyses are used such that the values of two characters are analyzed on a paired basis, results of which may be either positive or negative (Akhtar et al., 2015).

Studies on correlation coefficients of different characters are useful criterion to identify desirable traits that may contribute to improve the grain yield in QPM. Path analysis is also equally beneficial since it is an efficient biometrical tool which indicates the direct contribution of characters and its influence through other traits in influencing the yield (Nataraj et al., 2014). Generally, this method provides more information among variables than do correlation coefficients since this analysis provides the direct effects of specific yield components on yield, and indirect effects via other yield components (Garcia et al., 2003). Present study aimed at understanding the genetic parameters which determine the relationship between maize yield and other traits.

\section{Materials and Methods}

\section{Experimental material and experimental design}

The present investigation was carried out in Quality protein maize (Zea mays L.)"at Instructional farm, Rajasthan College of Agriculture, Maharana Pratap University of Agriculture and Technology, Udaipur, India during Kharif, 2014. The experimental material consisting of 22 genotypes presented in Table 1. In this experiment, 18 inbreeds and 4 checks viz., Pratap QPM hybrid-1, Vivek QPM-9, HQPM-1 and HQPM-5 were evaluated in randomized block design with three replications during Kharif 2014. The experimental material were sown in randomized block design with three replications with a single row plot of four meter length, maintaining crop geometry of $60 \times 25 \mathrm{~cm}$.

\section{Data collection}

Observations for all traits (anthesis silking interval, plant height, ear height, ear length, 100-grain weight, grain yield per plant, harvest index, oil content, starch content, protein content, tryptophan content and lysine content) were recorded on five randomly selected competitive plants of each entry in each replication except for days to 50 per cent tasseling, days to 50 percent silking and days to 75 per cent brown husk where observations were recorded on plot basis.

Estimation of oil content, starch content and protein content were done as per method suggested by Soxhlet's Ether Extraction method developed by A.O.A.C. (1965), Anthrone Reagent method and Micro kjeldahl's method given by Lindner (1944), respectively. Tryptophan was estimated through calorimetric method designed by Hernandez and Bates (1969). Lysine was estimated according to the calorimetric method by Villegas and Mertz (1971).

\section{Statistical analysis}

The phenotypic $\left(r_{p}\right)$, genotypic $\left(r_{g}\right)$ and environmental $\left(r_{e}\right)$ correlation coefficients for various characters were calculated by the method suggested by Panse and Sukhatme (1985). To establish a cause and effect relationship the first partition genotypic and phenotypic correlation coefficient into direct and indirect effects by path analysis as suggested by Dewey and Lu (1959) and developed by Wright (1921) than prepare path diagram based on cause and effect relationship. 


\section{Results and Discussion}

In the present investigation significant differences between the test entries were observed for all the yield and yield contributing traits and quality parameters presented in Table 2. The grain yield per plant had highly significant and positive correlations both at genotypic and phenotypic levels with 100-grain weight $\left(\mathrm{r}_{\mathrm{g}}=0.863\right.$, $\left.r_{p}=0.829\right)$, starch content $\left(r_{g}=0.657, r_{p}=0.649\right)$, harvest index $\left(r_{\mathrm{g}}=0.529, \mathrm{r}_{\mathrm{p}}=0.504\right)$, lysine Content $\left(r_{\mathrm{g}}=0.518, \mathrm{r}_{\mathrm{p}}=0.486\right)$, ear length $\left(r_{g}=0.476, \quad r_{p}=0.463\right)$, tryptophan content $\left(r_{g}=0.468, r_{p}=0.457\right)$ and ear height $\left(r_{g}=0.351\right.$, $\left.r_{p}=0.339\right)$ presented in Table 3. Whereas the anthesis silking interval $\left(\mathrm{r}_{\mathrm{g}}=-0.376, \mathrm{r}_{\mathrm{p}}=-0.256\right)$ had significant but negative correlation with seed yield at both the levels. Oil content $\left(\mathrm{r}_{\mathrm{g}}=-\right.$ $\left.0.376, r_{p}=-0.256\right)$ had non-significant and negative correlation at both the levels. Days to $50 \%$ tasseling $\left(r_{\mathrm{g}}=-0.121, \mathrm{r}_{\mathrm{p}}=-0.114\right)$, days to $75 \%$ brown husk $\left(r_{g}=-0.106, r_{p}=-0.102\right)$, days to $50 \%$ silking $\left(r_{\mathrm{g}}=-0.065, \mathrm{r}_{\mathrm{p}}=-0.063\right)$, plant height $\left(r_{\mathrm{g}}=-0.054, \mathrm{r}_{\mathrm{p}}=-0.046\right)$ and protein content $\left(r_{g}=-0.042, r_{p}=-0.043\right)$ had nonsignificant but positive correlation with seed yield per plant at both genotypic and phenotypic levels. Hence results were concluded that grain yield per plant had highly significant and positively correlated with 100-grain weight, starch content, harvest index, ear height and ear length at both the genotypic and phenotypic levels indicating that these attributes were more influencing the grain yield in maize and therefore, were important for bringing improvement in grain yield.

Most of the character pairs had higher values of genotypic correlations their corresponding phenotypic correlations. Such high amount of genotypic correlations could result due to masking or modifying effect of environmental on the association of characters. This indicates that though there was high degree of association between two variables at genotypic level, its phenotypic expression was deflated by the influence of environment. Days to 50 per cent tasseling had highly significant and positive association with days to 50 per cent silking and days to 75 per cent brown husk at both genotypic and phenotypic levels. Anthesis silking interval had highly significant but negative association with tryptophan Content, lysine content and 100grain weight. Plant height exhibited highly significant and positive association with ear height, ear length and lysine content. Ear height had highly significant and positive association with 100-grain weight, ear length and starch content. 100-grain weight had highly significant and positive association with harvest index, tryptophan content and lysine content. Harvest index had significant and positive association with oil content and tryptophan content. Protein content exhibited highly significant and positive association with tryptophan content.

The estimation of correlation coefficients indicates only the nature and extent of association between yield and yield attributes, but does not show the direct and indirect effects of different yield contributing attributes on yield per se. Grain yield is dependent on several characters which are mutually associated; these will in turn impair the true association exiting between various components and grain yield.

The genotypic correlation coefficients calculated for different pairs of characters were subjected to path coefficient analysis for partitioning these values into direct and indirect effects. The highest positive and direct effect was found for days to 50 per cent tasseling (5.559) followed by lysine content (0.710) and starch content (0.439). The negative and direct effect was found for days to 50 per cent silking (-5.774) and plant height $(-0.331)$ presented in Table 4. 
Table.1 Details of Inbred lines used

\begin{tabular}{llll}
\hline S.N. & Symbol/Code & \multicolumn{1}{c}{ Pedigree } & \multicolumn{1}{c}{ Origin } \\
\hline 1. & $\mathrm{~L}_{1}$ (EIQ-115) & NP-06-07R-58-3-2-1-2-1 & AICRP on maize, Udaipur \\
2. & $\mathrm{~L}_{2}$ (EIQ-116) & NP-06-07R-58-3-2-1-3-1 & AICRP on maize, Udaipur \\
3. & $\mathrm{~L}_{3}$ (EIQ-117) & NP-06-07R-74-19-1-1-1 & AICRP on maize, Udaipur \\
4. & $\mathrm{~L}_{4}$ (EIQ-118) & NP-06-07R-76-11-3-2-1 & AICRP on maize, Udaipur \\
5. & $\mathrm{~L}_{5}$ (EIQ-119) & NP-06-07R-76-9-1-1-2-2 & AICRP on maize, Udaipur \\
6. & $\mathrm{~L}_{6}$ (EIQ-120) & NP-06-07R-74-2-2-1-1 & AICRP on maize, Udaipur \\
7. & $\mathrm{~L}_{7}$ (EIQ-121) & NP-06-07R-77-1-2 & AICRP on maize, Udaipur \\
8. & $\mathrm{~L}_{8}$ (EIQ-122) & NP-06-07R-74-3-1-1-2-1 & AICRP on maize, Udaipur \\
9. & $\mathrm{~L}_{9}$ (EIQ-123) & NP-06-07R-74-3-1-1-3-2 & AICRP on maize, Udaipur \\
10. & $\mathrm{~L}_{10}$ (EIQ-124) & NP-06-07R-16-3 & AICRP on maize, Udaipur \\
11. & $\mathrm{~L}_{11}$ (EIQ-125) & NP-06-07R-76-8-3-3-1-8-2 & AICRP on maize, Udaipur \\
12. & $\mathrm{~L}_{12}$ (EIQ-126) & NP-06-07R-80-16-1-1-1-1-1 & AICRP on maize, Udaipur \\
13. & $\mathrm{~L}_{13}$ (EIQ-127) & NP-06-07R-89-12-1-1-1-1 & AICRP on maize, Udaipur \\
14. & $\mathrm{~L}_{14}$ (EIQ-128) & HO6R-6136-64-1-2-1-1 & CIMMYT \\
15. & $\mathrm{~L}_{15}$ (EIQ-129) & HO6R-64-1-4-1-1-2 & CIMMYT \\
16. & $\mathrm{~T}_{1}$ (EIQ-104) & NP-60 & AICRP on maize, Udaipur \\
17. & $\mathrm{~T}_{2}$ (EIQ-103) & NP-76 & AICRP on maize, Udaipur \\
18. & $\mathrm{~T}$ (EIQ-130) & NP-86 & AICRP on maize, Udaipur \\
19. & Pratap QPM & & AICRP on maize, Udaipur \\
& hybrid-1 & & \\
20. & Vivek QPM-9 & & VPKAS, Almora \\
21. & HQPM-1 & & CCS, HAU \\
22. & HQPM-5 & & CCS, HAU \\
\hline
\end{tabular}

Where,

AICRP - All India Coordinated Research Project

CSS HAU - Chaudhary Charan Singh Haryana Agricultural University

CIMMYT - International Wheat and Maize Improvement Centre

VPKAS - Vivekananda Parvatiya Krishi Anusandhan Sansthan, Almora

Table.2 Analysis of variance for fifteen traits

\begin{tabular}{llccc}
\hline SN & Source & Replication & Genotype & Error \\
\hline & & {$[2]$} & {$[21]$} & {$[42]$} \\
1 & Days to 50\% tasseling & $11.74^{* *}$ & $24.68^{* *}$ & 1.46 \\
2 & Days to 50\% silking & $10.29 * *$ & $24.70^{* *}$ & 1.35 \\
3 & Anthesis silking interval & 0.29 & $0.58^{* *}$ & 0.16 \\
4 & Days to 75\% brown husk & $14.02^{*}$ & $41.16^{* *}$ & 3.46 \\
5 & Plant height (cm) & 70.47 & $390.80^{* *}$ & 30.95 \\
6 & Ear height $(\mathrm{cm})$ & $171.92^{* *}$ & $981.06^{* *}$ & 23.78 \\
7 & Ear length $(\mathrm{cm})$ & $0.50^{* *}$ & $4.16^{* *}$ & 0.08 \\
8 & 100 Grain weight $(\mathrm{g})$ & 2.89 & $69.45^{* *}$ & 1.81 \\
9 & Grain yield per plant (g) & 0.80 & $1442.80^{* *}$ & 3.08 \\
10 & Harvest index (\%) & 0.42 & $20.63^{* *}$ & 0.51 \\
11 & Oil content $(\%)$ & 0.02 & $0.96^{* *}$ & 0.01 \\
12 & Starch content $(\%)$ & 0.05 & $21.67^{* *}$ & 0.14 \\
13 & Protein $(\%)$ & $0.01 *$ & $1.09^{* *}$ & 0.00 \\
14 & Tryptophan Content $(\%)$ & $0.0039^{* *}$ & $0.03^{* *}$ & 0.00 \\
15 & Lysine Content $(\%)$ & 0.02 & $0.33^{* *}$ & 0.01 \\
\hline
\end{tabular}


Table.3 Genotypic $(\mathrm{G})$ and phenotypic $(\mathrm{P})$ correlation coefficients among yield and quantitative traits in maize

\begin{tabular}{|c|c|c|c|c|c|c|c|c|c|c|c|c|c|c|c|c|}
\hline & & $\begin{array}{r}\text { Grain yield } \\
\text { per plant } \\
(\mathrm{g})\end{array}$ & \begin{tabular}{|r|} 
Days to \\
$50 \%$ \\
tasseling \\
\end{tabular} & $\begin{array}{r}\text { Days to } \\
50 \% \\
\text { silking } \\
\end{array}$ & $\begin{array}{r}\text { Anthesis } \\
\text { silking } \\
\text { interval } \\
\end{array}$ & $\begin{array}{r}\text { Days to } 75 \% \\
\text { brown husk }\end{array}$ & $\begin{array}{r}\text { Plant } \\
\text { height } \\
(\mathrm{cm})\end{array}$ & $\left.\begin{array}{r}\mathrm{Ear} \\
\text { height } \\
(\mathrm{cm})\end{array}\right]$ & $\begin{array}{r}\text { Ear } \\
\text { length } \\
(\mathrm{cm})\end{array}$ & $\begin{array}{c}100 \mathrm{Grain} \\
\text { weight }(\mathrm{g})\end{array}$ & $\begin{array}{r}\text { Harvest } \\
\text { index } \\
(\%) \\
\end{array}$ & $\begin{array}{r}\text { Oil } \\
\text { content } \\
(\%) \\
\end{array}$ & $\begin{array}{r}\text { Starch } \\
\text { content } \\
(\%) \\
\end{array}$ & $\begin{array}{r}\text { Protein } \\
(\%)\end{array}$ & $\begin{array}{r}\text { Tryptophan } \\
\text { Content }(\%)\end{array}$ & $\begin{array}{r}\text { Lysine } \\
\text { Content } \\
(\%) \\
\end{array}$ \\
\hline \multirow{2}{*}{$\begin{array}{l}\text { Grain yield per } \\
\text { plant }(\mathrm{g})\end{array}$} & $\mathrm{G}$ & 1.000 & 0.121 & 0.065 & -0.376 ** & 0.106 & 0.054 & $0.351 * *$ & $0.476^{* * *}$ & 0.863 *** & 0.529 *** & -0.175 & 0.657 *** & 0.042 & 0.468 *** & $0.518^{\text {*** }}$ \\
\hline & $\mathrm{P}$ & \begin{tabular}{|c|}
1.000 \\
\end{tabular} & 0.114 & 0.063 & $-0.2564 *$ & 0.102 & 0.046 & 0.339** & $0.463^{* *}$ & 0.829 *** & $0.504 * *$ & -0.173 & 0.649 *** & 0.043 & 0.457 *** & $0.486^{* * *}$ \\
\hline \multirow{2}{*}{$\begin{array}{l}\text { Days to } 50 \% \\
\text { tasseling }\end{array}$} & $\mathrm{G}$ & & 1.000 & 0.990 *** & 0.048 & 0.844 *** & \begin{tabular}{|l|}
-0.007 \\
\end{tabular} & -0.075 & 0.190 & 0.192 & 0.201 & \begin{tabular}{|c|}
-0.012 \\
\end{tabular} & 0.123 & 0.218 & 0.125 & -0.067 \\
\hline & $\mathrm{P}$ & & 1.000 & $0.983 * *$ & -0.062 & 0.759 ** & 0.065 & -0.068 & 0.152 & 0.158 & 0.173 & -0.008 & 0.103 & 0.206 & 0.116 & -0.064 \\
\hline \multirow{2}{*}{$\begin{array}{l}\text { Days to } 50 \% \\
\text { silking }\end{array}$} & $\mathrm{G}$ & & & 1.000 & 0.192 & 0.825 & -0.035 & -0.069 & \begin{tabular}{|l|}
0.192 \\
\end{tabular} & 0.142 & 0.198 & 0.031 & 0.093 & 0.175 & 0.038 & $\begin{array}{l}-0.104 \\
\end{array}$ \\
\hline & $\mathrm{P}$ & & & 1.000 & 0.115 & $0.745^{\text {*** }}$ & 0.048 & -0.065 & 0.154 & 0.118 & 0.162 & 0.032 & 0.074 & \begin{tabular}{|c|}
0.170 \\
\end{tabular} & 0.032 & -0.097 \\
\hline \multirow{2}{*}{$\begin{array}{l}\text { Anthesis silking } \\
\text { interval }\end{array}$} & $\bar{G}$ & & & & 1.000 & 0.019 & -0.217 & 0.072 & \begin{tabular}{|c|}
0.085 \\
\end{tabular} & $-0.306^{* *}$ & -0.060 & \begin{tabular}{|c|}
0.189 \\
\end{tabular} & \begin{tabular}{|c|}
-0.184 \\
\end{tabular} & $-0.321 * *$ & $-0.669 * *$ & $-0.327 * *$ \\
\hline & $\mathrm{P}$ & & & & 1.000 & -0.005 & -0.112 & 0.047 & 0.082 & $\begin{array}{r}-0.179 \\
\end{array}$ & \begin{tabular}{|l|}
-0.084 \\
\end{tabular} & 0.128 & \begin{tabular}{|c|}
-0.140 \\
\end{tabular} & -0.214 & $-0.481 * *$ & \begin{tabular}{|c|}
-0.215 \\
\end{tabular} \\
\hline \multirow{2}{*}{$\begin{array}{l}\text { Days to } 75 \% \\
\text { brown husk }\end{array}$} & $\overline{\mathrm{G}}$ & & & & & 1.000 & -0.060 & \begin{tabular}{|c|}
-0.049 \\
\end{tabular} & \begin{tabular}{|c|}
0.157 \\
\end{tabular} & 0.123 & 0.021 & $-0.266 * *$ & 0.161 & 0.049 & -0.124 & $-0.378^{* * *}$ \\
\hline & $\mathrm{P}$ & & & & & 1.000 & -0.040 & \begin{tabular}{|c|}
-0.031 \\
\end{tabular} & \begin{tabular}{|c|c|}
0.108 \\
\end{tabular} & \begin{tabular}{|c|}
0.101 \\
\end{tabular} & 0.004 & \begin{tabular}{|r|}
-0.234 \\
\end{tabular} & \begin{tabular}{|c|}
0.149 \\
\end{tabular} & 0.052 & \begin{tabular}{|c|}
-0.129 \\
\end{tabular} & $-0.268^{*}$ \\
\hline \multirow{2}{*}{ Plant height $(\mathrm{cm})$} & $\overline{\mathrm{G}}$ & & & & & & 1.000 & 0.413*** & $0.354 * *$ & \begin{tabular}{|c|}
0.195 \\
\end{tabular} & \begin{tabular}{|c|}
0.113 \\
\end{tabular} & 0.097 & 0.010 & -0.065 & 0.128 & $0.340^{* * *}$ \\
\hline & $\mathrm{P}$ & & & & & & 1.000 & $0.367 * *$ & $0.286 *$ & 0.182 & 0.118 & 0.091 & 0.000 & -0.058 & 0.098 & $0.251 *$ \\
\hline \multirow{2}{*}{ Ear height $(\mathrm{cm})$} & $\mathrm{G}$ & & & & & & & 1.000 & $0.546^{* *}$ & $0.609^{* * *}$ & 0.072 & $0.303 *$ & $0.515^{* * *}$ & -0.085 & $0.265 * *$ & 0.133 \\
\hline & P & & & & & & & 1.000 & $0.517 * *$ & $0.559 * *$ & 0.064 & $0.277 *$ & $0.498 * *$ & -0.077 & $0.244 *$ & 0.127 \\
\hline \multirow{2}{*}{ Ear length $(\mathrm{cm})$} & $\mathrm{G}$ & & & & & & & & 1.000 & $0.529 * *$ & $0.508^{* * *}$ & -0.043 & 0.065 & $-0.287 *$ & 0.218 & $0.399 * *$ \\
\hline & $\mathrm{P}$ & & & & & & & & 1.000 & $0.494 * *$ & $0.478^{\text {*** }}$ & -0.036 & 0.058 & $-0.282 *$ & 0.218 & $0.353^{* * *}$ \\
\hline \multirow{2}{*}{$\begin{array}{l}100 \text { Grain weight } \\
\text { (g) }\end{array}$} & $\mathrm{G}$ & & & & & & & & & 1.000 & 0.406 *** & \begin{tabular}{|l|}
-0.047 \\
\end{tabular} & 0.707 & 0.113 & $0.590^{* * *}$ & 0.627 *** \\
\hline & $\overline{\mathrm{P}}$ & & & & & & & & & \begin{tabular}{|c|}
1.000 \\
\end{tabular} & 0.400*** & \begin{tabular}{|c|}
-0.048 \\
\end{tabular} & $0.675^{* * * *}$ & 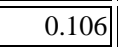 & $0.552 * *$ & 0.486*** \\
\hline \multirow{2}{*}{ Harvest index (\%) } & $\mathrm{G}$ & & & & & & & & & & 1.000 & $0.243^{*}$ & 0.100 & 0.096 & $0.298 *$ & $0.582^{* *}$ \\
\hline & $\mathrm{P}$ & & & & & & & & & & 1.000 & 0.227 & 0.090 & 0.089 & $0.274 *$ & $0.492^{* * *}$ \\
\hline \multirow{2}{*}{ Oil content $(\%)$} & $\mathrm{G}$ & & & & & & & & & & & 1.000 & 0.062 & 0.155 & 0.123 & 0.135 \\
\hline & $\mathrm{P}$ & & & & & & & & & & & 1.000 & 0.064 & 0.155 & 0.125 & 0.129 \\
\hline \multirow{2}{*}{ Starch content $(\%)$} & $\mathrm{G}$ & & & & & & & & & & & & 1.000 & 0.178 & 0.215 & 0.146 \\
\hline & $\mathrm{P}$ & & & & & & & & & & & & 1.000 & 0.176 & 0.205 & 0.140 \\
\hline \multirow{2}{*}{ Protein $(\%)$} & $\mathrm{G}$ & & & & & & & & & & & & & 1.000 & $0.325 * *$ & -0.093 \\
\hline & $\mathrm{P}$ & & & & & & & & & & & & & 1.000 & $0.322 * *$ & -0.082 \\
\hline \multirow{2}{*}{$\begin{array}{l}\text { Tryptophan } \\
\text { Content (\%) }\end{array}$} & $\mathrm{G}$ & & & & & & & & & & & & & & 1.000 & 0.674 *** \\
\hline & $\mathrm{P}$ & & & & & & & & & & & & & & 1.000 & $0.617^{* * * *}$ \\
\hline \multirow{2}{*}{ Lysine Content (\%) } & $\mathrm{G}$ & & & & & & & & & & & & & & & 1.000 \\
\hline & $\mathrm{P}$ & & & & & & & & & & & & & & & 1.000 \\
\hline
\end{tabular}




\section{Int.J.Curr.Microbiol.App.Sci (2017) 6(10): 2139-2146}

Table.4 Direct and indirect effect of characters on grain yield per plant $(\mathrm{g})$ at genotypic level

\begin{tabular}{|c|c|c|c|c|c|c|c|c|c|c|c|c|c|c|c|}
\hline & $\begin{array}{r}\text { Days to } \\
50 \% \\
\text { tasseling }\end{array}$ & $\begin{array}{r}\text { Days to } \\
50 \% \\
\text { silking }\end{array}$ & $\begin{array}{r}\text { Anthesis } \\
\text { silking } \\
\text { interval }\end{array}$ & $\begin{array}{r}\text { Days to } \\
75 \% \\
\text { brown } \\
\text { husk }\end{array}$ & $\begin{array}{r}\text { Plant } \\
\text { height } \\
(\mathrm{cm})\end{array}$ & $\begin{array}{r}\text { Ear } \\
\text { height } \\
(\mathrm{cm})\end{array}$ & $\begin{array}{r}\text { Ear } \\
\text { length } \\
(\mathrm{cm})\end{array}$ & $\begin{array}{r}100 \\
\text { Grain } \\
\text { weight } \\
(\mathrm{g})\end{array}$ & $\begin{array}{r}\text { Harvest } \\
\text { index } \\
(\%)\end{array}$ & $\begin{array}{r}\text { Oil } \\
\text { content } \\
(\%)\end{array}$ & $\begin{array}{r}\text { Starch } \\
\text { content } \\
(\%)\end{array}$ & $\begin{array}{r}\text { Protein } \\
(\%)\end{array}$ & $\begin{array}{l}\text { Tryptophan } \\
\text { Content }(\%)\end{array}$ & $\begin{array}{r}\text { Lysine } \\
\text { Content } \\
(\%)\end{array}$ & $\begin{array}{l}\text { Grain } \\
\text { yield per } \\
\text { plant }(\mathrm{g})\end{array}$ \\
\hline $\begin{array}{l}\text { Days to } 50 \% \\
\text { tasseling }\end{array}$ & 5.559 & 5.501 & 0.268 & 4.691 & -0.039 & -0.418 & 1.057 & 1.068 & 1.116 & -0.068 & 0.682 & 1.210 & 0.696 & -0.373 & 0.121 \\
\hline $\begin{array}{l}\text { Days to } 50 \% \\
\text { silking }\end{array}$ & -5.714 & -5.774 & -1.108 & -4.764 & 0.200 & 0.397 & -1.108 & -0.820 & -1.141 & -0.182 & -0.536 & -1.013 & -0.217 & 0.598 & 0.065 \\
\hline $\begin{array}{l}\text { Anthesis } \\
\text { silking } \\
\text { interval }\end{array}$ & 0.026 & 0.105 & 0.549 & 0.010 & -0.119 & 0.040 & 0.046 & -0.168 & -0.033 & 0.103 & -0.101 & -0.176 & -0.367 & -0.180 & $-0.376^{* *}$ \\
\hline $\begin{array}{l}\text { Days to } 75 \% \\
\text { brown husk }\end{array}$ & 0.233 & 0.228 & 0.005 & 0.276 & -0.017 & -0.014 & 0.043 & 0.034 & 0.006 & -0.074 & 0.045 & 0.014 & -0.034 & -0.105 & 0.106 \\
\hline $\begin{array}{l}\text { Plant height } \\
(\mathrm{cm})\end{array}$ & 0.002 & 0.012 & 0.072 & 0.020 & -0.331 & -0.137 & -0.117 & -0.065 & -0.037 & -0.032 & -0.003 & 0.022 & -0.042 & -0.113 & 0.054 \\
\hline $\begin{array}{l}\text { Ear height } \\
(\mathrm{cm})\end{array}$ & -0.028 & -0.026 & 0.027 & -0.018 & 0.153 & 0.372 & 0.203 & 0.226 & 0.027 & 0.113 & 0.191 & -0.032 & 0.098 & 0.050 & $0.351^{* * *}$ \\
\hline $\begin{array}{l}\text { Ear length } \\
(\mathrm{cm})\end{array}$ & 0.017 & 0.017 & 0.008 & 0.014 & 0.032 & 0.049 & 0.089 & 0.047 & 0.045 & -0.004 & 0.006 & -0.026 & 0.019 & 0.036 & $0.476^{* *}$ \\
\hline $\begin{array}{l}100 \text { Grain } \\
\text { weight }(\mathrm{g})\end{array}$ & -0.043 & -0.032 & 0.069 & -0.028 & -0.044 & -0.137 & -0.119 & -0.225 & -0.092 & 0.011 & -0.159 & -0.025 & -0.133 & -0.141 & $0.863 * *$ \\
\hline \begin{tabular}{|l|}
$\begin{array}{l}\text { Harvest index } \\
(\%)\end{array}$ \\
\end{tabular} & 0.059 & 0.058 & -0.018 & 0.006 & 0.033 & 0.021 & 0.149 & 0.119 & 0.293 & 0.071 & 0.029 & 0.028 & 0.087 & 0.170 & $0.529 * *$ \\
\hline $\begin{array}{l}\text { Oil content } \\
(\%)\end{array}$ & 0.003 & -0.007 & -0.043 & 0.061 & -0.022 & -0.070 & 0.010 & 0.011 & -0.056 & -0.230 & -0.014 & -0.036 & -0.028 & -0.031 & -0.175 \\
\hline $\begin{array}{l}\text { Starch } \\
\text { content }(\%)\end{array}$ & 0.054 & 0.041 & -0.081 & 0.071 & 0.004 & 0.226 & 0.028 & 0.311 & 0.044 & 0.027 & 0.439 & 0.078 & 0.094 & 0.064 & $0.657 * *$ \\
\hline Protein $(\%)$ & 0.030 & 0.024 & -0.044 & 0.007 & -0.009 & -0.012 & -0.040 & 0.016 & 0.013 & 0.022 & 0.025 & 0.138 & 0.045 & -0.013 & 0.042 \\
\hline \begin{tabular}{|l|l}
$\begin{array}{l}\text { Tryptophan } \\
\text { Content }(\%)\end{array}$ \\
\end{tabular} & -0.029 & -0.009 & 0.153 & 0.028 & -0.029 & -0.061 & -0.050 & -0.135 & -0.068 & -0.028 & -0.049 & -0.075 & -0.229 & -0.154 & $0.468 * *$ \\
\hline $\begin{array}{l}\text { Lysine } \\
\text { Content }(\%)\end{array}$ & -0.048 & -0.074 & -0.232 & -0.269 & 0.242 & 0.095 & 0.283 & 0.445 & 0.413 & 0.096 & 0.104 & -0.066 & 0.479 & 0.710 & $0.518^{* *}$ \\
\hline
\end{tabular}


The days to 50 per cent tasseling showed highly positive indirect effect for days to 50per cent silking (5.501) followed by days to 75 per cent brown husk (4.691) and harvest index (1.116). Whereas, it has found negative indirect effect for ear height (-0.418) followed by lysine content (-0.373) and oil content (0.068). The 100 grain weight showed positive indirect effect for anthesis silking interval (0.069) followed by oil content (0.011) and highly negative indirect effect was found for starch content $(-0.159)$ followed by ear height $(-0.137)$ and tryptophan content $(-0.133)$.

These findings were in agreement with reports of Alok kumar et al., (1999), Venugopal et al., (2003) for plant height and ear length; Kumar et al., (2006), Shakoor et al., (2007), Sofi and Rather (2007) and Saidaiah et al., (2008) for 100 grain weight; Venugopal (2003), Kumar et al., (2006) and Brar et al., (2008) for ear height and Kumar et al., (2006) for days to $50 \%$ tasseling.

The success of a breeding program depends upon the genetic variation in the materials at a hand. The high direct effects of these characters appeared to be the main reason for their strong association with grain yield. Hence, direct selection for these characters would be very effective. As such these results will be useful for choosing populations to be used in developing new maize populations with improved characters.

\section{References}

A.O.A.C. 1965. "Official methods for oil analysis for association of Official Agricultural Chemists" $10^{\text {th }}$ Ed. Washington., D.C.

Akhtar, L.H., R. Minhas, M.S. Bukhari and S.A.S. Shah. 2015. Genetic Analysis of Some Quantitative Traits in Cluster Bean (Cyamopsis tetragonoloba L.). Journal of Environmental \&
Agricultural Sciences. 4:48-51.

Alok Kumar, Gangashetti, M.G. and Dahia, A. 1999. Analysis of direct and indirect effects for quantitative traits in diallel crosses of maize (Zea mays L.). Annals of Biology, Ludhiana 15(2):173-176.

Brar, S.P.S., Chawla, J.S and Singh, P. 2008. Studies on different selection indices and path analysis in maize (Zea mays L.). Crop Improvement 35(1): 16-19.

Dewey, D.R., and Lu, H.K. 1959. A correletion and path coefficient analysis of components of creasted wheat grass and seed production. Agronomy Journal 51: 515-518.

Downsell, C.R., Paliwal, R.L. and Cantrell, R.P. 1996. Maize in the third world. West view press, pp. 1-37.

FAO. 2014. World Agricultural Production http://faostat.fao.org/default.aspx. Accessed 14 April. 2013.

Garcia, L.F., Rharrabti, Y., Villegas, D. and Royo, C. 2003. Evaluation of grain yield and its components in durum wheat under Mediterranean conditions. An Ontogenic Approach. Agron. J. 95: 266-274.

Hernandez, H.H., and Bates, L.S. 1969. A modified method for rapid tryptophan analysis in maize. International maize and wheat improvement center research bulletin, 13: 1-7.

Khatab, I.A., Farid M.A. and Kumamaru, T. 2016. Genetic diversity associated with heading date in some rice (Oryza sativa L.) genotypes using microsatellite markers. Journal of Environmental \& Agricultural Sciences. 6: 58-63.

Kumar, S., Shahi, J.P., Singh, J. and Singh, S.P. 2006. Correlation and path analysis inearly generation inbreds of maize (Zea mays L.). Crop Improvement 33(2): 156-160.

Linder, R.C., 1944. Rapid analytical method for some of the more common inorganic constituents of plant tissues. Plant 
Physiology. 19: 76-89.

Nataraj, V., Shahi, J. P. and Agarwal, V. 2014. Correlation and Path Analysis in Certain Inbred Genotypes of Maize (Zea Mays L.) at Varanasi. International journal of innovative research \& development. 3(1): 14-17.

Pandey, Y., Vyas, R.P., Kumar, J., Singh, L., Singh, H.C., Yadav, P.C. and Vishwanath 2017. Heritability, Correlation and Path Coefficient Analysis for Determining Interrelationships among Grain Yield and Related Characters in Maize (Zea mays L.). Int. J. Pure App. Biosci. 5(2): 595-603.

Panse, V.G., and Sukhatme, P.V. 1985. Statistical methods for agricultural workers, ICAR Publication, New Delhi, pp.145.

Saidaiah, P., Satyanarayana, E. and Kumar, S.S. 2008. Association and path coefficient analysis in maize (Zea mays L.). Agricultural Science Digest 28: 7983.

Shakoor, M.S., Muhammadand, A. and Hussain, A. 2007. Correlation and path analysis studies in maize double crosses. Pak. J. Agri. Sci., 44(2): 213216.

Sofi, P.A., and Rather, A.G. 2007. Studies on genetic variability, correlation and path analysis in maize (Zea mays L.). MaizeGenetics-Cooperation-Newsletter (81): 26-27.

Venugopal, M., Ansari, N.A. and Rajanikanth, T. 2003. Correlation and path analysis in maize (Zea mays L.). Crop Res., Hisar, 25(3): 525-529.

Villegas, E., and Mertz, E.T. 1971. Chemical screening methods for quality protein maize. Research Bulletin No. 20, CIMMYT.

\section{How to cite this article:}

Ramesh Kumar, R.B. Dubey, K.D. Ameta, Ram Kunwar, Rajani Verma and Prashant Bisen. 2017. Correlation and Path Coefficient Analysis for Yield Contributing and Quality Traits in Quality Protein Maize (Zea mays L.). Int.J.Curr.Microbiol.App.Sci. 6(10): 2139-2146. doi: https://doi.org/10.20546/ijcmas.2017.610.253 